\title{
ARTICLE
}

\section{Scintillation Efficiency of Inorganic Scintillators for Intermediate-Energy Charged Particles}

\author{
Yusuke KOBA $^{1^{*}}$, Hiroki IWAMOTO ${ }^{1}$, Katashi KIYOHARA $^{1}$, Toshinori NAGASAKI ${ }^{1}$, \\ Genichiro WAKABAYASHI ${ }^{1}$, Yusuke UOZUMI ${ }^{1}$, Naruhiro MATSUFUJI ${ }^{2}$ \\ ${ }^{I}$ Department of Applied Quantum Physics and Nuclear Engineering, \\ Kyushu University, 744 Motooka Nishi-ku, Fukuoka 812-0395, Japan \\ ${ }^{2}$ National Institute of Radiological Sciences, 4-9-1 Anakawa Inage-ku, Chiba 263-8555, Japan
}

\begin{abstract}
We carried out experiments to investigate the light output response of $\mathrm{NaI}(\mathrm{Tl}), \mathrm{CsI}(\mathrm{Tl}), \mathrm{GSO}(\mathrm{Ce})$ and $\mathrm{LYSO}(\mathrm{Ce})$ crystals for intermediate-energy ${ }^{4} \mathrm{He},{ }^{12} \mathrm{C}$ and ${ }^{40} \mathrm{Ar}$ beams from HIMAC at National Institute of Radiological Sciences. And we investigate the light output of these crystals for several-energy gamma-ray for comparison. From these light output responses, we obtained the relationships between the scintillation efficiency (dL/dx) and the specific energy loss $(\mathrm{dE} / \mathrm{dx})$ for each crystal. The scintillation efficiency curves of $\mathrm{NaI}(\mathrm{Tl})$ and $\mathrm{CsI}(\mathrm{Tl})$ crystal have the peak in a particular $\mathrm{dE} / \mathrm{dx}$. On the other hand, the scintillation efficiency curves of $\mathrm{GSO}(\mathrm{Ce})$ and $\mathrm{LYSO}(\mathrm{Ce})$ crystal decrease with increasing $\mathrm{dE} / \mathrm{dx}$. The light output curves of these crystals were systematically reproduced using obtained scintillation efficiencies.
\end{abstract}

KEYWORDS: inorganic scintillator, scintillation efficiency, specific energy loss, $\mathrm{NaI}(\mathrm{Tl}), \mathrm{CsI}(\mathrm{Tl}), \mathrm{GSO}(\mathrm{Ce})$, LYSO (Ce)

\section{Introduction}

It is well known that the light output response of inorganic scintillator bear a nonlinear relationship with the deposited energy. In order to improve the energy identification performance, it is necessary for this nonlinearity to be investigated enough.

In high energy experiments, various scintillators have been developed strenuously with the advancement of energy and beam intensity of accelerators. The performance requested for these scintillators is heavier, faster and more tolerant to radiation damage. $\mathrm{GSO}(\mathrm{Ce})$ and $\mathrm{LYSO}(\mathrm{Ce})$ are one of the scintillators which have been developed for these requests. In order to use them as high precision calorimeters, it is necessary to investigate the light output characteristics of these scintillators. Especially, it is very important to define a light output curve which is the relationship between the energy deposited in crystals and the amount of the luminescence.

We carried out experiments on the light output characteristics of several scintillators for intermediate-energy (several hundred $\mathrm{MeV} / \mathrm{u}$ ) charged particles in order to investigate these nonlinear relationships in wide energy range $^{1)}$. Focusing attention on the relationship between the scintillation efficiency $(\mathrm{dL} / \mathrm{dE})$ which is the amount of the luminescence per unit energy, and the specific energy loss $(\mathrm{dE} / \mathrm{dx})$, we investigated the light output response of four kinds of crystals, $\mathrm{NaI}(\mathrm{Tl}), \mathrm{CsI}(\mathrm{Tl}), \mathrm{GSO}(\mathrm{Ce})$, and $\mathrm{LYSO}(\mathrm{Ce})$. And we investigate the light output of these crystals for several-energy gamma-ray for comparison. The light output curves of these crystals were systematically reproduced.

*Corresponding author; (tel)092-802-3479, (fax)092-802-3479, (e-mail)yusemi@nucl.kyushu-u.ac.jp

\section{Experiment}

Measurements were performed at the PH1 course of HIMAC (Heavy Ion Medical Accelerator in Chiba) in the National Institute of Radiological Sciences. Monochromatic ${ }^{4} \mathrm{He},{ }^{12} \mathrm{C}$ and ${ }^{40} \mathrm{Ar}$ particles accelerated up to $180 \mathrm{MeV} / \mathrm{u}, 290$ $\mathrm{MeV} / \mathrm{u}$ and $650 \mathrm{MeV} / \mathrm{u}$ were used in the experiments. And Monochromatic proton $(80 \mathrm{MeV})$ was used in the $\operatorname{LYSO}(\mathrm{Ce})$ experiment. Figure 1 shows the schematic view of present measurement system. It was composed of, from upstream, a degrader changer, an active collimator, a thin plastic scintillator with the thickness of $2 \mathrm{~mm}$, and two inorganic scintillators. The active collimator has an aperture of $5 \mathrm{~mm}$ diameter as an active slit to determine the solid angle of the spectrometer. The thin plastic scintillator generated the trigger signals by the coincidence method with the signal of the inorganic crystal. The dimensions of inorganic scintillators are $\mathrm{NaI}(\mathrm{Tl}): 50.8 \mathrm{~mm}, \mathrm{CsI}(\mathrm{Tl}): 43 \mathrm{~mm}$, $\mathrm{GSO}(\mathrm{Ce}): 43 \mathrm{~mm}$, and $\mathrm{LYSO}(\mathrm{Ce}): 20 \mathrm{~mm}$ cube. Each crystal was coupled to a photomultiplier tube (Hamamatsu R329: $\mathrm{NaI}(\mathrm{Tl}), \mathrm{R} 580$ : $\mathrm{CsI}(\mathrm{Tl})$ and $\mathrm{GSO}(\mathrm{Ce}), \mathrm{R} 7415$ : LYSO(Ce) ). In order to change incident energy to the inorganic scintillators we used some degraders made of aluminum or copper. The measured energy region was $150 \sim 700 \mathrm{MeV}$ for ${ }^{4} \mathrm{He}, 500 \sim 3,400 \mathrm{MeV}$ for ${ }^{12} \mathrm{C}, 4,000$ $\sim 14,000 \mathrm{MeV}$ for ${ }^{40} \mathrm{Ar}$. The light output of scintillators was normalized with a LED and ${ }^{137} \mathrm{Cs}$ radiation sources. The unit of each light output is $\left[1 / \mathrm{L}\left({ }^{137} \mathrm{Cs}\right)\right]$, where $\mathrm{L}\left({ }^{137} \mathrm{Cs}\right)$ is the light output of each crystal for $0.662 \mathrm{MeV}$ gamma.

The deposited energy in crystals or degraders by charged particles was calculated by the following equation, 


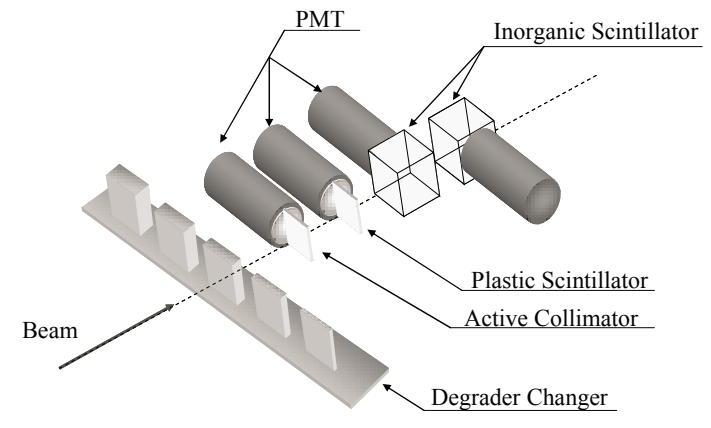

Fig.1 The schematic view of present measurement system. Each scintillator was attached a photomultiplier tube.

$-\frac{d E}{d x}=4 \pi r_{0}^{2} z^{2} \frac{m_{e} c^{2}}{\beta^{2}} N Z\left[\ln \left(\frac{2 m_{e} c^{2}}{I}\right)-\ln \left(1-\beta^{2}\right)-\beta^{2}\right]$,

where $r_{0}$ is the classical electron radius, $z$ is charge of incident particles, $m_{e} c^{2}$ is the rest mass energy of electron, $Z$ is atomic number of material, $N$ is atomic density of material, $I$ is the mean excitation potential. In the measured energy range Eq. (1) calculates the specific energy loss with satisfactory accuracy.

\section{RESULT}

Figure 2 shows the light output of scintillators as a function of deposit energy for several charged particles and gamma ray. In Figure 2 error bar shows the standard deviation of total absorption peaks. The larger the atomic number of incident particle is, the smaller the light output of the same deposited energy is.
As described above, the light output curves considerably change its shape with the measured particle. Therefore it is difficult that the light output (L) is represented by the unified equation with the deposited energy (E). So we focused attention on the relationship between the scintillation efficiency $(\mathrm{dL} / \mathrm{dE})$ and the specific energy loss $(\mathrm{dE} / \mathrm{dx})$. At first, the scintillation efficiency was defined approximate slope of the light output curve on each measurement. The specific energy loss on incident particles without electrons was calculated with Eq. (1). In low dE/dx field, the electrons were generated by converting the gamma rays to the energetic electrons through photoelectric effect in crystals. The specific energy loss of electrons was referred to ESTAR $^{2)}$. Figures 3 $\sim \mathbf{6}$ show the relationship between the scintillation efficiency and the specific energy loss of crystals $^{3,4)}$. In all region of the specific energy loss, the scintillation efficiency of $\mathrm{GSO}(\mathrm{Ce})$ and $\mathrm{LYSO}(\mathrm{Ce})$ decreases monotonically, but that of $\mathrm{NaI}(\mathrm{Tl})$ and $\mathrm{CsI}(\mathrm{Tl})$ become lowering in the region of the low specific energy loss.

\section{DISCUSSION}

In order to represent the light output curves of organic scintillators, Birks showed the following equation on the assumption that the amount of the luminescence per unit length is described by a function of its stopping power,

$$
\frac{d L}{d x}=\frac{S(d E / d x)}{1+k B(d E / d x)}
$$

where $S$ is the absolute scintillation efficiency and $k B$ is the Birks parameter. Eq. (2) shows that the amount of the luminescence per unit length decreases by the quenching

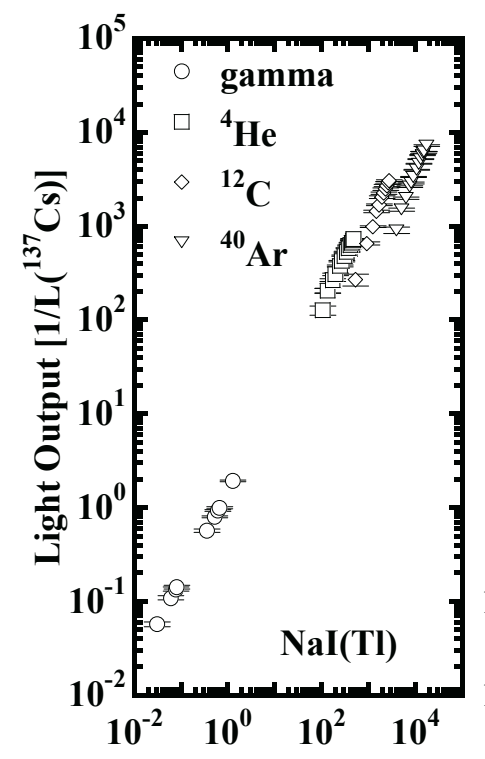

Energy Deposit [MeV]

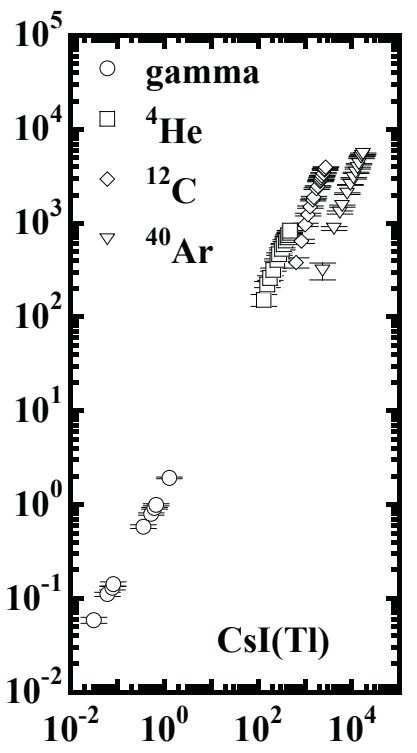

Energy Deposit [MeV]
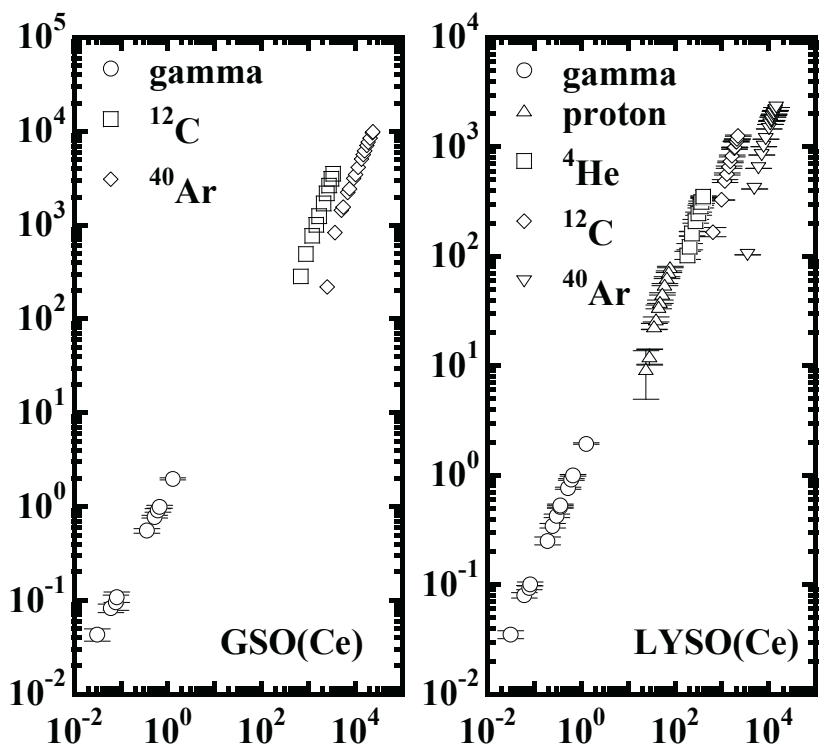

Energy Deposit [MeV]

\section{Energy Deposit [MeV]}

Fig.2 The light output of $\mathrm{NaI}(\mathrm{Tl}), \mathrm{CsI}(\mathrm{Tl}), \mathrm{GSO}(\mathrm{Ce})$ and $\mathrm{LYSO}(\mathrm{Ce})$ crystal as a function of deposit energy to gamma and several charged particles. 


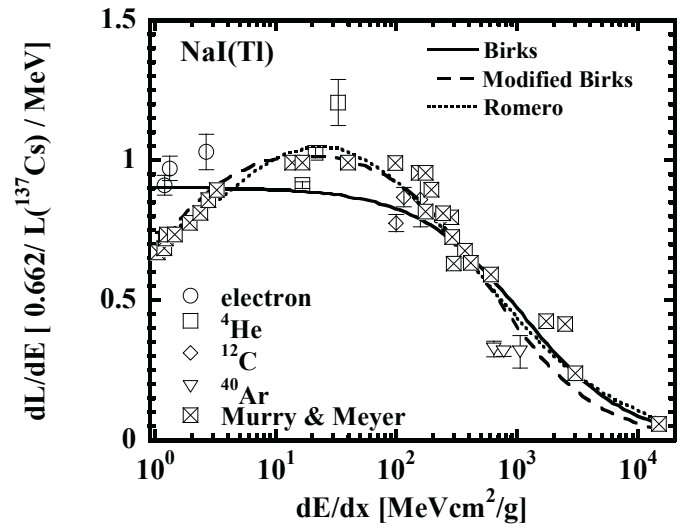

Fig.3 Scintillation Efficiency of $\mathrm{NaI}(\mathrm{Tl})$.

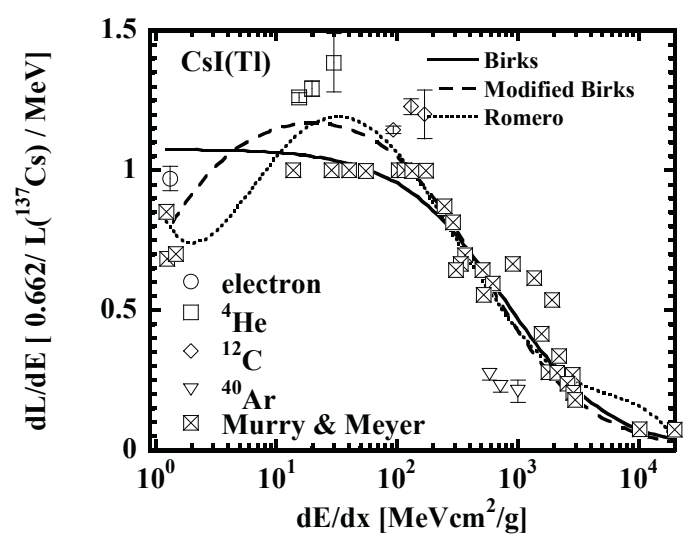

Fig.4 Scintillation Efficiency of CsI(Tl).

effect in high $\mathrm{dE} / \mathrm{dx}$ region. Eq. (2) reproduced light output curves of organic scintillators appropriately.

The equation of scintillation efficiency $(\mathrm{dL} / \mathrm{dE})$ is derived by Eq. (2),

$$
\frac{d L}{d E}=\frac{a}{1+b(d E / d x)} .
$$

And in order to reproduce quenching in low specific energy loss range, we modify Eq. (3) as,

$$
\frac{d L}{d E}=\frac{a}{1+b(d E / d x)+c(d E / d x)^{-1}} .
$$

The scintillation efficiency of $\mathrm{NaI}(\mathrm{Tl})$ for several charged particles was reported by Romero et al. ${ }^{5)}$. Romero et al. parameterized $\mathrm{dL} / \mathrm{dE}$ as a function of $\mathrm{dE} / \mathrm{dx}$ in the following way,

$$
\frac{d L}{d E}=\sum_{t=0}^{5} a_{t}\left(\ln \frac{d E}{d x}\right)^{t} .
$$

In order to represent the scintillation efficiency of these crystals, we applied the above equations. Table 1 shows parameters for equations obtained with best fit. Because the scintillation efficiency curves of $\mathrm{NaI}(\mathrm{Tl})$ and $\mathrm{CsI}(\mathrm{Tl})$ have quenching in the region of low specific energy loss, these curves are not represent well by Birks equation, which shows monotonically decreasing function. Figure 7 shows the light output curves calculated using Eq. (1) or ESTAR

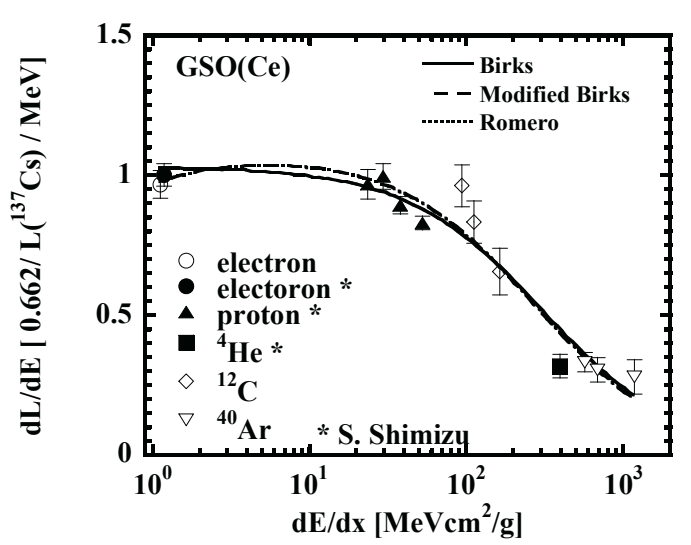

Fig.5 Scintillation Efficiency of GSO(Ce).

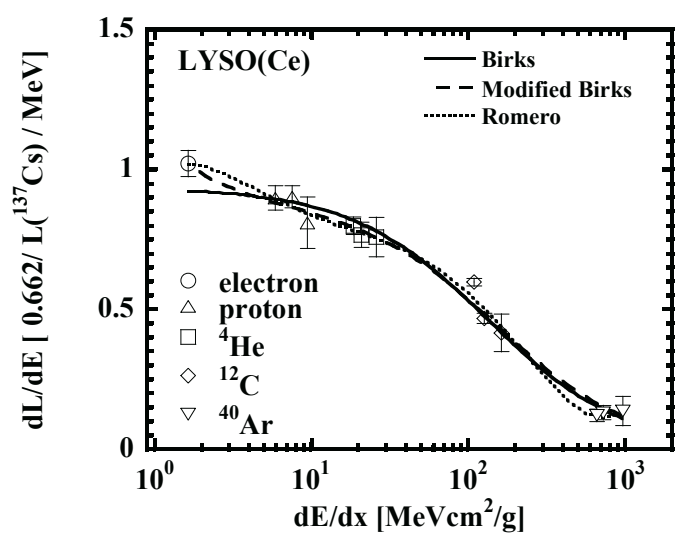

Fig.6 Scintillation Efficiency of LYSO(Ce).

and Eq. (4) with parameters of Table 1. These calculation curves reproduced the light output response of each crystal roughly using simple equation, despite the kinds of the particle are different.

Table 1 The parameters for each equation.

\begin{tabular}{rcccc}
\hline & $\mathrm{NaI}(\mathrm{Tl})$ & $\mathrm{CsI}(\mathrm{Tl})$ & $\mathrm{GSO}(\mathrm{Ce})$ & $\mathrm{LSYO}(\mathrm{Ce})$ \\
\hline Birks & & & & \\
$\mathrm{a}$ & 0.905 & 1.08 & 1.03 & 0.938 \\
$\mathrm{~b}$ & $9.10 \times 10^{-4}$ & $1.29 \times 10^{-3}$ & $3.22 \times 10^{-3}$ & $7.60 \times 10^{-3}$ \\
Modified & & & & \\
Birks & & & \\
$\mathrm{a}$ & 1.08 & 1.26 & 1.08 & 0.883 \\
$\mathrm{~b}$ & $1.65 \times 10^{-3}$ & $1.92 \times 10^{-3}$ & $3.66 \times 10^{-3}$ & $6.50 \times 10^{-3}$ \\
$\mathrm{c}$ & 0.594 & 0.747 & 0.110 & -0.241 \\
& & & & \\
Romero & & & & \\
$\mathrm{a}_{0}$ & 0.765 & 0.912 & 0.897 & 0.975 \\
$\mathrm{a}_{1}$ & $2.76 \times 10^{-2}$ & -0.535 & 0.707 & 0.219 \\
$\mathrm{a}_{2}$ & 0.104 & 0.514 & -0.667 & -30.7 \\
$\mathrm{a}_{3}$ & $-3.81 \times 10^{-2}$ & -0.143 & 0.243 & 0.124 \\
$\mathrm{a}_{4}$ & $4.09 \times 10^{-3}$ & $1.52 \times 10^{-3}$ & $-3.89 \times 10^{-2}$ & $-2.19 \times 10^{-2}$ \\
$\mathrm{a}_{5}$ & $-1.42 \times 10^{-4}$ & $-5.59 \times 10^{-4}$ & $2.22 \times 10^{-3}$ & $1.35 \times 10^{-3}$ \\
\hline
\end{tabular}




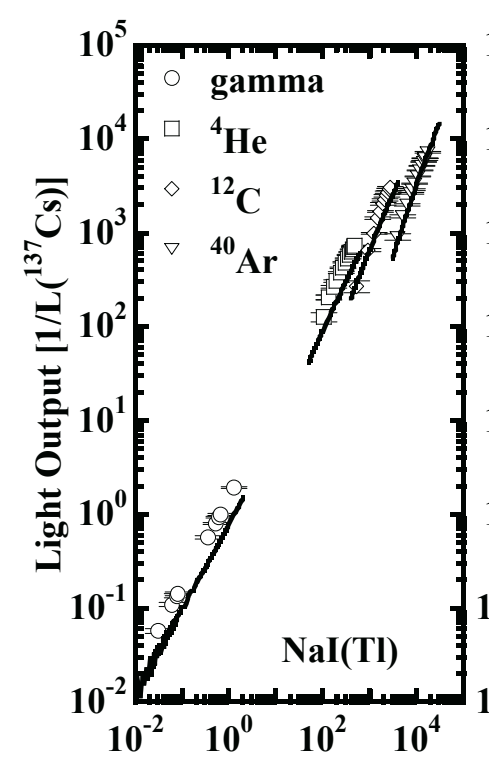

Energy Deposit [MeV]

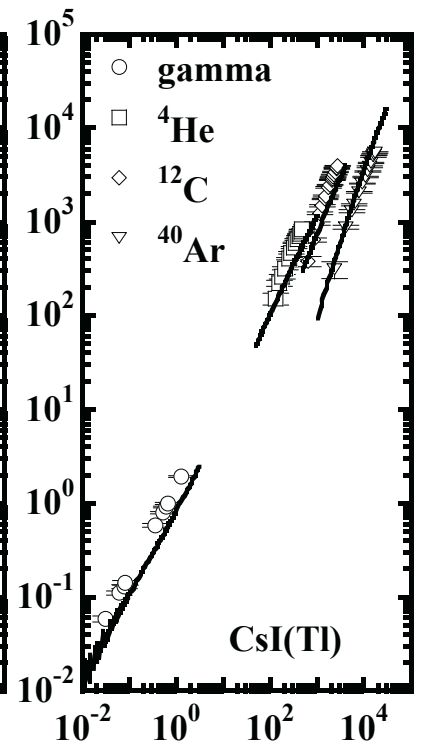

Energy Deposit $[\mathrm{MeV}]$

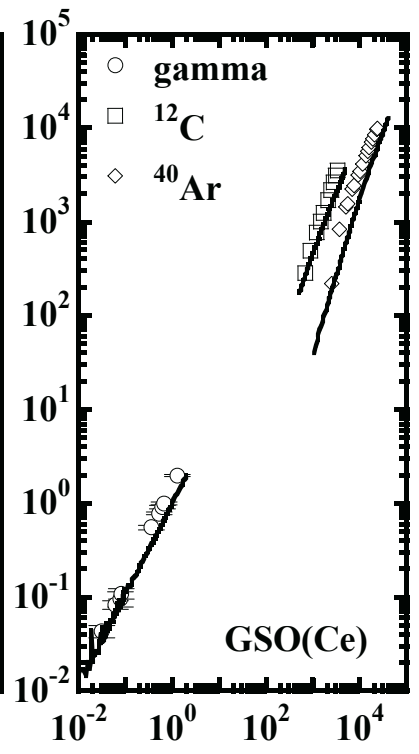

Energy Deposit [MeV]

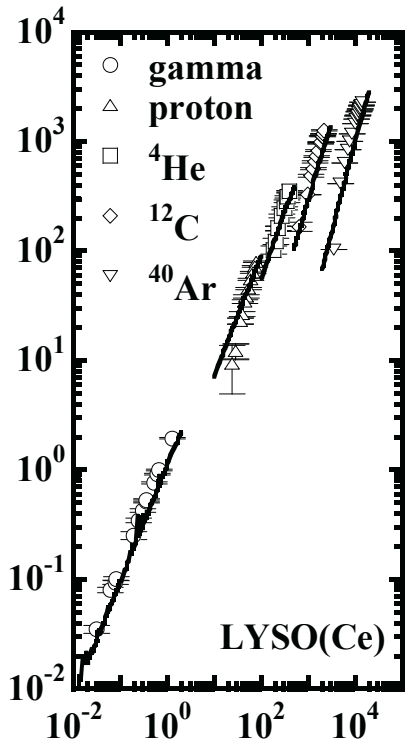

Energy Deposit [MeV]

Fig.7 The calculation curves of the light output.

\section{CONCLUSION}

In order to investigate the light output response of inorganic scintillators, we carried out experiments at PH1 course of HIMAC (Heavy Ion Medical Accelerator in Chiba) in National Institute of Radiological Sciences.

By measurements of the wide range of intermediate energy, the scintillation efficiency for the wide range of the specific energy loss was measured. The scintillation efficiency of each crystal could be represented by some simple equation independent on the kind of particles. And the calculation curves using an equation could represent the light output of these crystals roughly. This study shows that the scintillation efficiency is a function of the specific energy loss. The more accurate scintillation efficiency is represented, the more strictly the light output curve may be represented. It is anticipated that the more refined researches of scintillation efficiency clear up the light output characteristics of inorganic scintillators.

\section{ACKNOWLEDGEMENT}

The authors are grateful to staff members of the National
Institute of Radiological Sciences. This work was performed as the Research Project with Heavy Ions at the NIRS-HIMAC, 18 P 214, 20 P 239.

\section{REFERENCES}

1) F. Saiho, T. Kin et al., "Response and efficiency of stacked $\mathrm{GSO}(\mathrm{Ce})$ spectrometer to intermediate-energy deuterons", Nucl. Instr. \& Meth. A 537, 594 (2005).

2) M.J. Berger, J.S. Coursey, M.A. Zucker and J. Chang, "Stopping-Power and Range Tables for Electrons, Protons, and Helium Ions, ESTAR",

(http://physics.nist.gov/PhysRefData/Star/Text/contents.html)

3) R. B. Murray and A. Meyer, "Scintillation Response of Activated Inorganic Crystals to Various Charged Particles", Phys. Rev. Vol.122-3, 815 (1961).

4) S. Shimizu, M. Suzuki, T. Koizumi et al., "Light output and decay curve of GSO:Ce under electron, proton, alpha, particle and fission fragment excitations", Nucl. Instr. \& Meth. A 537, 57 (2005).

5) J. L. Romero, G. A. Needham et al., "The response of $\mathrm{NaI}(\mathrm{Tl})$ to 30-60 MeV Z=1 particles", Nucl. Instr. \& Meth. A 301, 241 (1991). 